\title{
Significado psicológico de la violencia y la agresión en una muestra urbana colombiana
}

\section{Psychological meaning of violence and aggression in a colombian urban sample}

\author{
Irene Salas-Menotti* \\ Corporación Universitaria \\ Iberoamericana, Bogotá, Colombia \\ Recibido: 8 de abril de 2008 \\ Revisado: 28 de mayo de 2008 \\ Aceptado: 26 de junio de 2008
}

\section{Resumen}

El objetivo de esta investigación fue identificar el significado psicológico de la violencia y la agresión en una muestra urbana colombiana, así como determinar los elementos distintivos que guían a los individuos en la construcción de estos conceptos. Asimismo, se buscó identificar los elementos, conductas, sentimientos o emociones implicados en la valoración de la violencia y la agresión. Participaron 485 hombres y mujeres, con edades comprendidas entre los 12 y los 74 años, y se utilizó la Técnica de Redes Semánticas Naturales Modificadas. Se encontró que ambos conceptos tienen considerables elementos compartidos, lo cual genera el uso indistinto de estos términos en el lenguaje coloquial, sin embargo, también tienen suficientes elementos diferenciadores para ser percibidos como constructos diferentes.

Palabras clave: violencia, agresión, significado psicológico, redes semánticas.

\footnotetext{
* Correspondencia: Irene Salas-Menotti. Facultad de Psicología, Universidad Santo Tomás. Correo electrónico: irenesalasmenotti@gmail.com. Dirección postal: Cra. 9 No. 51-11, Bogotá, Colombia. Proyecto Docente \# 957, Facultad de Psicología, Corporación Universitaria Iberoamericana. La autora agradece la colaboración de los siguientes estudiantes de la Facultad de Psicología de la Corporación Universitaria Iberoamericana en la recolección de los datos: Maritza Ardila, Lina Becerra, Sonia Castañeda, Fernando Cristancho, Ángela Herrera, Andrea León, Leonardo Luna, Diana Moreno, Adriana Quimbayo, Adriana Rodríguez, Carolina Sánchez.
} 


\section{Abstract}

The main objective of this study was to identify the psychological meaning of violence and aggression in a Colombian urban sample, as well as to determine the similarities and differences regarding age and gender. 485 men and women participated, with ages ranging from 12 trough 74 years. The Modified Natural Semantic Networks technique was used and it established that individuals are able to distinguish violence from aggression on the basis of the affective and behavioral responses typically associated to each one of them, nonetheless, the two concepts overlap in their psychological meaning, and they are used indifferently in everyday language.

Key words: violence, aggression, psychological meaning, semantic networks.

\section{Introducción}

A lo largo de la revisión de la literatura científica, tanto del concepto de violencia como el de agresión, se encuentran traslapes entre sí. Algunas definiciones consideran la violencia como daño físico; este hecho no la distingue de las definiciones más difundidas de agresión. A pesar de esta confusión teórica, se reconoce que existen ambos conceptos, el problema radica en que no hay un estudio sistemático que aporte algo nuevo al conocimiento de la violencia que no sea a partir de observaciones conductuales o mediciones físicas de recursos (Domínguez, 2003, Domínguez, Reyes Lagunes \& Múzquiz, 2003).

En el ámbito de la Psicología se ha abordado el estudio de la violencia desde diferentes perspectivas: su prevención, su impacto en la dinámica familiar, la violencia en el ámbito de pareja, su relación con el consumo de alcohol, etc. Este interés por estudiar la violencia recalca que es uno de los problemas de más relevancia en los últimos tiempos, sin embargo, no todas las investigaciones realizadas (ver Domínguez, 2003) comparten la misma concepción de violencia, como se esperaría en una misma disciplina. De ahí se desprende la preocupación teórica de cómo se está conceptualizando este fenómeno.

Si bien el lenguaje no sólo revela las conductas específicamente culturales, sino que también se da a la labor de etiquetarlas (Adair, 1994), y la interpretación de un constructo o situación puede variar según el significado cultural que se dé a dicho concepto (Holtzman, 1994), la mayoría de los estudios realizados toman como pauta in- vestigaciones o teorías elaboradas en los Estados Unidos, pero si se entiende que la agresión y la violencia, cualquiera que sea su concepción, se da en el plano de las relaciones interpersonales, las cuales tienen in situ el elemento de la interpretación por parte de una de las partes, se encuentra que su estudio tampoco estaría exento de las influencias culturales. De tal manera se tendría la obligación de encontrar si las concepciones teóricas son totalmente aplicables al contexto cultural colombiano.

En el Diccionario de la Real Academia Española (2007), el término violencia hace alusión, por una parte, al efecto de violentarse que, a su vez, es estar fuera del estado natural de proceder $y$, por otra, a actuar con fuerza. Designa fuerza o impetuosidad temperamental, la cual no necesariamente es natural, sino también inducida. En cualquier caso, lleva una intención o propósito dirigido hacia algo o alguien (Conrado, 1970). Al hablar de violencia, entonces, se hace referencia a algo o alguien que está fuera de su estado natural, que obra con ímpetu o fuerza, y que se dirige a un objetivo con la intención de forzarlo. Puede ser física y verbal, puede emplear la amenaza, la persecución o la intimidación (García, 1999) como formas de ejercicio del poder, y se va imponiendo como forma para resolver los conflictos o reclamar los derechos ciudadanos (Rosario, 1999).

En el Diccionario de la Real Academia Española (2007) se propone que la palabra agresión es el efecto de agredir que, a su vez, hace referencia a acometer contra alguien para matarlo, herirlo o hacerle cualquier daño. La agresión es "una conducta destructiva o punitiva dirigida ha- 
cia una persona u objeto" (Corsini, 1999, p. 29). Es un fenómeno multifactorial que tiene muchos determinantes y sirve para distintos propósitos. Este constructo es generalmente definido como una conducta que resulta en un daño personal. Sin embargo, no todos los daños son considerados como agresivos. Para que un acto sea evaluado como agresivo o no, depende de los juicios subjetivos de intencionalidad y de causalidad.

Como se puede observar en las definiciones anteriores, es evidente una confusión, tanto teórica como en el lenguaje coloquial, con respecto a lo que cada palabra significa. Como es sabido, los sistemas simbólicos usados por los individuos en la construcción del significado son sistemas profundamente enraizados en la cultura y su lenguaje, de forma tal que se constituyen en un conjunto comunal de herramientas especiales que convierte a quien los usa en un reflejo de su comunidad.

Para la medición del significado se han utilizado diversas técnicas como el diferencial semántico (Osgood, 1952), las asociaciones libres (Szalay \& Bryson, 1973; 1974) y las representaciones sociales (Moscovici, 1984). Estos modelos conforman la base de la teoría de las redes semánticas. Esta aproximación establece que la información contenida en la memoria a largo plazo está organizada en forma de redes, en las cuales las palabras forman relaciones, que como conjuntos dan significado a un concepto y se convierten, de esta manera, en un método del estudio del significado.

En esta línea se encuentran Figueroa, González y Solís (1981), quienes propusieron que el estudio del significado debería ser a través de redes semánticas naturales, es decir, que se deben analizar respuestas generadas directamente por las personas. La red semántica de un concepto es aquel conjunto de palabras elegidos por la memoria a través de un proceso reconstructivo que no sólo está dada por los vínculos asociativos, sino también por la naturaleza de los procesos de memoria que eligen los elementos que la integran. Dicha selección no se hace basándose en la fuerza de la asociación, sino en la clase de propiedades de los elementos que integran la red.
Desde esta perspectiva, los objetivos principales de este estudio fueron: 1) conocer el significado psicológico que de los conceptos violencia y agresión tienen hombres y mujeres, de diferentes edades, de una muestra urbana colombiana, 2) identificar si éstos difieren significativamente entre sí, y 3) determinar si los significados son semejantes o diferentes dependiendo del sexo y la edad.

\section{Método}

\section{Participantes}

A través de muestreo no probabilístico por cuotas participaron 485 personas ( 242 hombres y 243 mujeres) divididos en cuatro grupos de edad: adolescentes, jóvenes, adultos y adultos mayores.

\section{Técnica}

Se exploraron los constructos de interés a partir de la Técnica de Redes Semánticas Naturales Modificadas (RSNM) (Reyes-Lagunes, 1993), cuyo objetivo principal fue obtener el significado psicológico con base en los núcleos de la red y los elementos más próximos a cada concepto. Esta técnica es útil para el estudio de la representación y utilización de la información en la memoria, usada para la determinación del significado de un concepto (Figueroa, González \& Solís, 1981). Mediante esta técnica se puede obtener una idea clara de los aspectos sobresalientes, dirección, profundidad e intensidad generales de los constructos que se están investigando, así como aspectos culturales y conductuales. Los mismos autores explican que la estructura semántica se va desarrollando y adquiriendo nuevas relaciones y elementos a medida que aumenta el conocimiento general del individuo, a este respecto se puede considerar que las influencias del medio, así como los años de experiencia van enriqueciendo y transformando el significado de los conceptos.

\section{Instrumento y procedimiento}

Se construyó un instrumento formado por cuatro hojas tamaño esquela, en el que se encontraban 
los estímulos en la parte superior. La primera hoja contenía las instrucciones y el cuestionario demográfico. En las hojas restantes se pusieron, en forma aleatoria, los estímulos violencia, agresión y televisión (que fue utilizado a manera de distractor), en la mayoría de los casos, éste fue aplicado en forma colectiva a los sujetos, y el tiempo promedio empleado fue de 15 minutos.

Se les dieron instrucciones a los sujetos para que escribieran, al menos cinco palabras sueltas (sustantivos, adjetivos, verbos y adverbios) por cada estímulo. Posteriormente, se les pidió que jerarquizaran las palabras de acuerdo con la importancia que cada palabra tuviera con respecto al estímulo, de manera que se le asignara el número uno a la palabra más importante, el número dos a la segunda palabra más importante y, así sucesivamente hasta terminar la lista.

\section{Tratamiento estadístico}

Con base en el protocolo de las RSNM, primero se obtuvo el número total de palabras definidoras o tamaño de red (TR), por grupos de edad y por sexo, para cada estimulo. Después se obtuvieron las sumas de las frecuencias ponderadas para cada palabra o peso semántico (PS). El núcleo de la red (NR) simboliza el máximo de palabras asociado a cada estímulo, sin que se pierda representativi- dad de significado. La distancia semántica cuantitativa (DSC) se obtuvo asignando el porcentaje más alto $(100 \%)$ a aquella definidora que resultaba más cercana a cada uno de los estímulos y se realizó una reglas de tres para determinar el valor de las palabras restantes. También se obtuvo el índice de consenso grupal o valor ICG, que permitió la identificación de convergencias o disparidades por grupos, tanto por sexo y edad, en cuanto al uso de palabras definidoras de cada estímulo. Este índice representa el porcentaje que corresponde a las palabras que comparten cada grupo y sexo.

Luego de obtener cada uno de estos índices se recurrió a la utilización de algunas pruebas estadísticas que permitieron dar sustento a algunos hallazgos. En primer lugar se utilizó la prueba $X^{2}$ para probar si existía alguna diferencia entre grupos de acuerdo con el TR.

\section{Resultados}

Todos los análisis se realizaron para los dos estímulos de interés. Cada valor obtenido se calculó por grupo de edad y por sexo. En las tablas 1 y 2 se presenta el número de definidoras asociadas para cada estímulo por grupos de edad y sexo. En las sumatorias totales se excluyen las palabras repetidas por todos los grupos.

Tabla 1. Tamaño de Red (TR) por grupos de edad y sexo

\begin{tabular}{|c|c|c|c|c|c|c|c|c|c|c|c|c|}
\hline \multirow{3}{*}{ TR } & \multicolumn{12}{|c|}{ Edad } \\
\hline & \multicolumn{3}{|c|}{ 13-18 años } & \multicolumn{3}{|c|}{ 19-30 años } & \multicolumn{3}{|c|}{ 31-55 años } & \multicolumn{3}{|c|}{$56+$ años } \\
\hline & $F$ & M & Total & $\mathrm{F}$ & M & Total & $F$ & M & Total & $\mathrm{F}$ & M & Total \\
\hline Violencia & 116 & 106 & 162 & 170 & 188 & 289 & 89 & 120 & 121 & 95 & 150 & 200 \\
\hline Agresión & 121 & 126 & 171 & 171 & 187 & 284 & 97 & 140 & 193 & 91 & 136 & 167 \\
\hline
\end{tabular}

Tabla 2. Tamaño de red (TR) por sexo

\begin{tabular}{cccc}
\hline TR & F & M & Total \\
\hline Violencia & 293 & 439 & 486 \\
Agresión & 311 & 408 & 526 \\
\hline
\end{tabular}

Se compararon los TR por grupos de edad y sexo con la prueba de $\mathrm{X}^{2} \mathrm{y}$, como se puede observar en las tablas 3 y 4 , se encontró que existen diferencias significativas en el número de palabras que los participantes originaron para los dos estímulos, tanto en los diferentes grupos de edad, como por sexo. 
Tabla 3. Valores $X^{2}$ para tamaño de red violencia

\begin{tabular}{lll}
\hline \multicolumn{1}{c}{${\text { Prueba } \mathrm{x}^{2}}$} & \multicolumn{1}{c}{ Edad } & \multicolumn{1}{c}{ Sexo } \\
\hline $\mathrm{X}^{2}$ & $79,84^{*}$ & $29,12^{*}$ \\
$\mathrm{Gl}$ & 3 & 1 \\
$\mathrm{a} .01$ & 11.345 & 6.635 \\
\hline
\end{tabular}

Tabla 4. Valores $X^{2}$ para tamaño de red agresión

\begin{tabular}{ccc}
\hline Prueba $\mathrm{x}^{2}$ & Edad & Sexo \\
\hline $\mathrm{X}^{2}$ & $44,07^{*}$ & $12,66^{*}$ \\
$\mathrm{Gl}$ & 3 & 1 \\
$\mathrm{a} .01$ & 11.345 & 6.635 \\
\hline
\end{tabular}

El grupo de edad que marcó la diferencia, debido a que produjo significativamente más palabras, fue el de 19 a 30 años, mientras que fueron los hombres los que produjeron más palabras en total. Esto quiere decir que estos grupos, al producir una mayor cantidad de palabras, tienen los conceptos de violencia y agresión más difusos, por lo que les cuesta trabajo definirlos de manera limitada (Domínguez, 2003; Salas-Menotti, 2003; Valdez, 1998). Por el contrario, los grupos que produjeron un menor número de definidoras, para el caso de violencia, fueron las mujeres de 31 a 45 años, y para el caso de agresión, las mujeres mayores de 56 años. Estos resultados indican que las experiencias que tienen los participantes de diferentes grupos de edad y sexo, en este caso, son definitivos para determinar la riqueza de la red y, asimismo, la vaguedad o precisión de los conceptos.

Cuando se hace la comparación del número de palabras totales elicitadas entre los dos estímulos, se encuentra que sólo el grupo de adultos de 31 a 55 años presenta una diferencia significativa en el número de palabras generadas para cada concepto; en este caso el concepto agresión fue el que marcó la diferencia. Sin embargo, al hacer la comparación entre las redes totales de cada estímulo no se observó una diferencia significativa, aunque nuevamente el estímulo agresión tuvo un tamaño de red mayor. Esto lleva a pensar que a la población general le cuesta más trabajo definir lo que es agresión, que violencia.
La DSC representa la distancia que existe entre los conceptos, a partir de la definidora de mayor PS que representa el $100 \%$ de toda la red semántica. En las siguientes figuras se presentan las redes semánticas de cada uno de los estímulos; en éstas se puede observar que en el estímulo violencia, la definidora agresión se encuentra en lugar prioritario dentro del núcleo de red. Lo mismo sucede con el concepto agresión, es decir, que frente a este estímulo se encuentra la definidora violencia dentro del NR. Esto indica que para la muestra total, e incluso para las submuestras por edad y sexo, los dos conceptos están muy relacionados; sin embargo, se observa que las palabras asociadas a cada red difieren en cualidad para cada uno de los estímulos, por grupo y sexo.

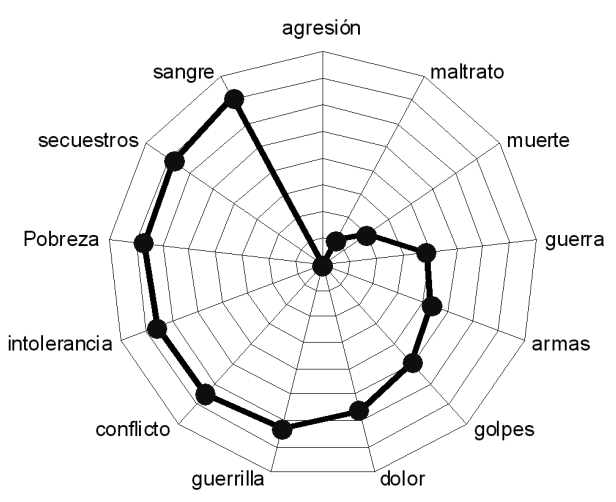

Figura 1. Distancia semántica cuantitativa del estímulo violencia en la muestra total

En el caso del estímulo violencia (figura 1), se puede observar que es definida en primer lugar con la palabra agresión, seguida de maltrato, muerte y guerra. Vale la pena resaltar que en el núcleo de red de este concepto aparecen palabras relacionadas con el conflicto armado colombiano como guerrilla, conflicto, armas y secuestros. De igual forma, se observa que la violencia es definida con palabras que hacen referencia a fenómenos que podrían ser su causa como pobreza e intolerancia.

En relación con el estímulo agresión (figura 2), se encontró que es definido, en primer lugar, con la palabra maltrato, seguido de la palabra golpes. Al igual que sucedió con el estimulo anterior, se ob- 
serva que la definidora violencia aparece dentro del núcleo de red en un lugar importante. Se encontró que la mayoría de palabras que conforman el NR son expresiones observables de la agresión, como peleas, groserías, insultos y gritos; también se evidencian los distintos tipos de agresión: la verbal y la física.

Cuando se comparan los núcleos de red de los dos conceptos se puede observar que comparten las siguientes palabras: dolor, golpes, intolerancia y maltrato. Esto permite señalar que, aunque los dos conceptos se parecen, e incluso pueden llegar a ser confundidos, cada uno cuenta con elementos que los distinguen y los diferencian claramente en el lenguaje de la muestra elegida.

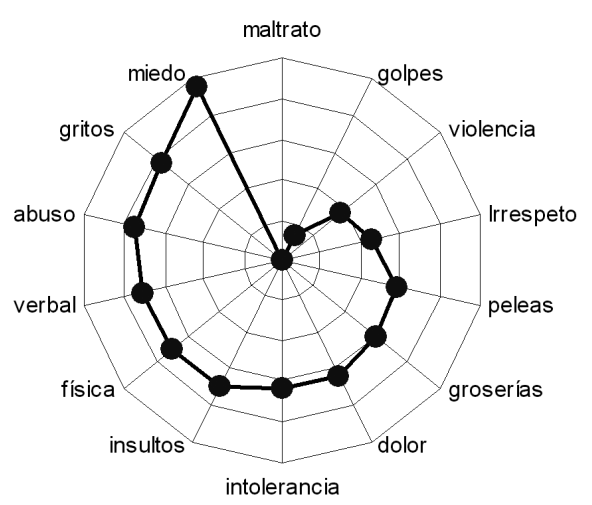

Figura 2. Distancia semántica cuantitativa del estímulo agresión en la muestra total

A continuación se presentan los Índices de Consenso Grupal (ICG), los cuales, como se mencionó anteriormente, representan el porcentaje que corresponden a las palabras que comparten los participantes de cada grupo.

Tabla 5. Índice de Consenso Grupal por grupos de edad

\begin{tabular}{ccccc}
\hline \multicolumn{5}{c}{ Edad } \\
\hline \multirow{2}{*}{ ICG } & $\begin{array}{c}13-18 \\
\text { años }\end{array}$ & $\begin{array}{c}19-30 \\
\text { años }\end{array}$ & $\begin{array}{c}31-55 \\
\text { años }\end{array}$ & $56+$ años \\
\hline \multirow{2}{*}{ Violencia } & $33,7 \%$ & $47,3 \%$ & $62 \%$ & $17,8 \%$ \\
Agresión & $38,98 \%$ & $21,35 \%$ & $17,9 \%$ & $31,21 \%$ \\
\hline
\end{tabular}

Si se observa el ICG obtenido por los grupos de edad se puede observar que los grupos de 19 a 30 años y de 31 a 55 años presentan IGC relativamente altos para el estimulo violencia, ya que en estudios similares (Domínguez, 2003) los IGC no superaron el $28 \%$; este hallazgo permite decir que el concepto de violencia se encuentra bien definido y que está más o menos afianzado en estos grupos en particular. También se observa que el estímulo agresión presentó los IGC más bajos en los grupos de jóvenes y adultos, lo que indica que el concepto de agresión es difuso y no está del todo afianzado. Este mismo fenómeno se puede observar en el grupo de adultos mayores, pero en relación con la violencia.

Tabla 6. Índice de Consenso Grupal por sexo

\begin{tabular}{lcc}
\hline \multicolumn{1}{c}{ ICG } & Mujeres & Hombres \\
\hline Violencia & $6,3 \%$ & $6,57 \%$ \\
Agresión & $5,16 \%$ & $4,68 \%$ \\
\hline
\end{tabular}

Si se observan los ICG por sexo se aprecia que son bajos, comparados con los grupos de edad. Esto indica que los hombres y las mujeres tienen una concepción diferente, y a la vez le dan un significado diferente a cada uno de los conceptos de interés. Las experiencias que hombres y mujeres tienen con los fenómenos de violencia y agresión difieren en cantidad y cualidad por lo que se asocian con conductas, emociones, evaluaciones diferentes, y la construcción que se hace de cada uno de ellos depende de estas experiencias.

Tabla 7. Índice de Consenso Grupal global

\begin{tabular}{lc}
\hline \multicolumn{1}{c}{ ICG } & General \\
\hline Violencia & $3,17 \%$ \\
\hline Agresión & $1,28 \%$ \\
\hline
\end{tabular}

Al determinar el ICG de toda la muestra se observa que es bastante bajo, lo que indica que los conceptos son difusos y no están claramente definidos en el lenguaje cotidiano de la muestra total. Los significados, al estar determinados en gran medida por las experiencias en relación con éstos, pueden presentar una gran variabilidad si 
las experiencias que tienen los individuos de una sociedad difieren en intensidad, cantidad, tipo de exposición, etc. Evidentemente, es diferente el concepto de violencia que tiene una persona de 80 años que ha vivido en carne propia el conflicto armado colombiano, al de una persona de 15 años que vive el conflicto por televisión y que está en constante contacto con otros tipo de violencia simbólicos como, por ejemplo, la que se observa en los juegos de computador.

De igual manera, el hecho de que los ICG sean bajos puede ser indicio de que tanto la violencia como la agresión se han convertido en fenómenos cotidianos, por lo tanto, son más difíciles de definir de manera concreta y focalizada.

Tabla 8. Palabras compartidas por todos los grupos

\begin{tabular}{|cc|}
\hline VIOLENCIA & AGRESIÓN \\
\hline Agresión & Maltrato \\
\hline Dolor & Violencia \\
\hline Maltrato & Dolor \\
\hline Guerra & Verbal \\
\hline Golpes & Irrespeto \\
\hline Conflicto & Ofensa \\
\hline Intolerancia & Groserías \\
\hline Pobreza & \\
\hline Sufrimiento & \\
\hline Sangre & \\
\hline Maldad & \\
\hline Irrespeto & \\
\hline Soledad & \\
\hline Odio & \\
\hline Robo & \\
\hline
\end{tabular}

En la tabla anterior se presentan las palabras compartidas por todos los grupos. A simple vista se puede percibir que las redes de violencia y agresión comparten varias palabras en común: dolor, maltrato, irrespeto, violencia y agresión (éstas fueron excluidas cuando eran el estímulo), lo cual indica que los dos conceptos tienen redes muy parecidas y esto permitiría explicar el porqué de la confusión que existe en el lenguaje cotidiano y del que surge el uso indistinto de éstas. Sin embargo, también se puede advertir que cada una de las redes tiene elementos que las identifican claramente de las otras.
Para afinar aún más el estudio, se obtuvieron correlaciones bivariadas para datos ordinales (rho ( $\rho)$ de Spearman-Brown) por las submuestras analizadas anteriormente, en aras de conocer la correspondencia de las jerarquías que ocupan las palabras definidoras comunes en cada uno de los estímulos. A continuación, se muestran, a través de tablas, sólo aquellas correlaciones que resultaron significativas al .01.

En la tabla 9 se presentan las correlaciones para los grupos de edad del estímulo violencia, y se puede observar que todas las correlaciones fueron significativas, lo cual indica que las redes generadas por los diferentes grupos de edad son similares, siendo las redes más parecidas las de los grupos de adolescentes y ancianos, y las menos parecidas las generadas por los grupos de adolescentes y adultos. Estos hallazgos llaman la atención, ya que se esperaría mayor similaridad entre grupos de edad adyacentes, por la cercanía en experiencias e historia.

En la tabla 10 se observan las correlaciones para los grupos de edad del estímulo agresión, y se puede observar que casi todas las correlaciones, excepto aquélla entre adolescentes y ancianos, fueron significativas, lo cual indica que las redes generadas por los diferentes grupos de edad son similares, siendo las redes más parecidas las de los grupos de adolescentes y jóvenes y las de jóvenes y adultos.

Las correlaciones por sexos (tabla 11) resultaron ser significativas para los dos estímulos, lo que implica que tanto hombres como mujeres asignan de manera similar la importancia de cada definidora en la red.

En las tablas 12 y 13 se pueden observar las correlaciones para los estímulos violencia y agresión por grupos de edad y sexo. En el caso del estímulo violencia se observa que casi todas las correlaciones, excepto aquélla entre las mujeres adolescentes y las mujeres adultas, resultaron ser significativas; esto indica que en las redes semánticas evocadas por los participantes hay alta concordancia, en cuanto a la jerarquía que les dan a 
las palabras definidoras en las que convergen las redes. Aunque la mayoría de correlaciones resultaron ser significativas, se observó que las redes de mujeres y hombres jóvenes y las de mujeres y hombres adultos resultaron ser más similares entre sí, ya que comparten un mayor número de palabras, comparados con los demás grupos. Las redes que resultaron menos similares fueron aquéllas producidas por los grupos de mujeres adolescentes y ancianas.

Tabla 9. Coeficiente $\rho$ significativo de Spearman-Brown por grupos de edad para el estímulo violencia

\begin{tabular}{ccccc}
\hline \multicolumn{5}{c}{ Grupo de edad } \\
\hline & $13-18$ años & $19-30$ años & $31-55$ años & $56+$ años \\
\hline \multirow{2}{*}{ 13-18 años } & -- &, 732 &, 615 &, 619 \\
& & $\mathrm{n}=60$ & $\mathrm{n}=44$ & $\mathrm{n}=56$ \\
$19-30$ años & -- &, 655 &, 731 \\
& & $\mathrm{n}=61$ & $\mathrm{n}=72$ \\
31-55 años & & -- &, 593 \\
56+ años & & & $\mathrm{n}=56$ \\
& & & -- \\
\hline
\end{tabular}

Tabla 10. Coeficiente $\rho$ significativo de Spearman- Brown por grupos de edad para el estímulo agresión

\begin{tabular}{ccccc}
\hline \multicolumn{5}{c}{ Grupo de edad } \\
\hline & $13-18$ años & $19-30$ años & $31-55$ años & $56+$ años \\
\hline \multirow{2}{*}{ 13-18 años } & - &, 374 &, 529 & \\
& & $\mathrm{n}=70$ & $\mathrm{n}=56$ &, 366 \\
19-30 años & - & $\mathrm{n}=72$ & $\mathrm{n}=59$ \\
& & - & $\mathrm{n}=50$ \\
31-55 años & & & - \\
\hline
\end{tabular}

Tabla 11. Coeficiente $\rho$ significativo de SpearmanBrown por sexos para los estímulos violencia y agresión

\begin{tabular}{cc}
\hline & $\rho$ \\
\hline Violencia &, 791 \\
& $\mathrm{n}=220$ \\
Agresión &, 692 \\
& $\mathrm{n}=167$ \\
\hline
\end{tabular}

En las correlaciones para el estímulo agresión (tabla 13) por grupos de edad y sexo se puede observar que de las 28 correlaciones posibles,
19 resultaron significativas. Es importante mencionar que las redes generadas por los hombres jóvenes no se correlacionaron con aquéllas producidas por los adolescentes hombres y mujeres. De igual manera, las redes generadas por las mujeres ancianas no se correlacionaron con aquéllas producidas por los adolescentes hombres y los jóvenes hombres y mujeres. Asimismo, las redes originadas por los hombres ancianos no se correlacionaron con las redes de mujeres adolescentes y adultas ni con las redes de los hombres jóvenes. Esto indica que la jerarquización que cada uno de estos grupos realiza es diferente, aunque compartan cierto número de palabras. 
Tabla 12. Coeficiente $\rho$ significativo de Spearman- Brown por grupos de edad y sexo para el estímulo violencia

\begin{tabular}{|c|c|c|c|c|c|c|c|c|c|}
\hline & & \multicolumn{2}{|c|}{ 13-18 años } & \multicolumn{2}{|c|}{ 19-30 años } & \multicolumn{2}{|c|}{ 31-55 años } & \multicolumn{2}{|c|}{$56+$ años } \\
\hline & & $M$ & $\mathrm{H}$ & M & $\mathrm{H}$ & M & $\mathrm{H}$ & M & $\mathrm{H}$ \\
\hline \multirow{3}{*}{ 13-18 años } & \multirow{2}{*}{ M } & \multirow{2}{*}{ - } & \multirow{2}{*}{, $70 n=50$} & \multirow{2}{*}{,432 n=40 } & 426 & & ,366 & ,506 & \multirow{2}{*}{, $429 n=40$} \\
\hline & & & & & $n=40$ & & $n=35$ & $n=25$ & \\
\hline & $\mathrm{H}$ & & - & ,605 n=42 & $\begin{array}{l}, 518 \\
n=41\end{array}$ & $\begin{array}{l}, 467 \\
n=32\end{array}$ & $\begin{array}{l}, 605 \\
n=34\end{array}$ & $\begin{array}{l}, 533 \\
n=31\end{array}$ &, $460 n=35$ \\
\hline \multirow{4}{*}{ 19-30 años } & \multirow{2}{*}{ M } & & & \multirow{4}{*}{ - } & ,707 & ,609 & ,592 &, 574 & \multirow{2}{*}{, $647 n=50$} \\
\hline & & & & & $n=107$ & $n=49$ & $n=54$ & $n=36$ & \\
\hline & \multirow{2}{*}{$\mathrm{H}$} & & & & & 662 & 686 & ,598 & \multirow{2}{*}{,625 n=49 } \\
\hline & & & & & - & $n=47$ & $n=51$ & $n=40$ & \\
\hline \multirow{3}{*}{ 31-55 años } & \multirow{2}{*}{ M } & & & & & 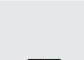 & ,862 &, 553 & \multirow{2}{*}{, $731 n=40$} \\
\hline & & & & & & & $n=76$ & $n=37$ & \\
\hline & H & & & & & & - & $\begin{array}{l}, 365 \\
n=39\end{array}$ &, $715 n=42$ \\
\hline \multirow{3}{*}{$56+$ años } & M & & & & & & & - &, $561 n=36$ \\
\hline & & & & & & & & & \\
\hline & $\mathrm{H}$ & & & & & & & & _ \\
\hline
\end{tabular}

Tabla 13. Coeficiente $\rho$ significativo de Spearman- Brown por grupos de edad y sexo para el estímulo agresión

\begin{tabular}{|c|c|c|c|c|c|c|c|c|c|}
\hline \multicolumn{10}{|c|}{ Grupo de edad } \\
\hline & & \multicolumn{2}{|c|}{ 13-18 años } & \multicolumn{2}{|c|}{ 19-30 años } & \multicolumn{2}{|c|}{ 31-55 años } & \multicolumn{2}{|c|}{$56+$ años } \\
\hline & & M & $\mathrm{H}$ & M & $\mathrm{H}$ & M & $\mathrm{H}$ & M & $\mathrm{H}$ \\
\hline \multirow{4}{*}{ 13-18 años } & $M$ & & ,654 & $476 n=41$ & & ,453 & ,640 & ,519 & \multirow{4}{*}{, $466 n=35$} \\
\hline & in & - & $n=67$ & 1, & & $\mathrm{n}=35$ & $n=37$ & $n=29$ & \\
\hline & $\mathrm{H}$ & & - &, $563 n=40$ & & & ,552 & & \\
\hline & & & & & & & $\mathrm{n}=38$ & & \\
\hline \multirow{4}{*}{ 19-30 años } & M & & & & ,682 & ,458 & ,722 & & \multirow{4}{*}{,461 n=4 } \\
\hline & & & & & $n=60$ & $n=42$ & $\mathrm{n}=39$ & & \\
\hline & $\mathrm{H}$ & & & & & ,395 & 494 & & \\
\hline & & & & & - & $n=42$ & $n=42$ & & \\
\hline \multirow{4}{*}{ 31-55 años } & $M$ & & & & & & & ,465 & \multirow{3}{*}{, $563 n=29$} \\
\hline & & & & & & - & & $n=33$ & \\
\hline & $\mathrm{H}$ & & & & & & 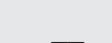 & ,469 & \\
\hline & $M$ & & & & & & & $n=30$ & $636 n=51$ \\
\hline $56+$ años & $\mathrm{H}$ & & & & & & & - & , \\
\hline
\end{tabular}

En estas correlaciones significativas se observó que las redes de mujeres y hombres adolescentes resultaron ser más similares entre sí, ya que comparten un mayor número de palabras, compa- rados con los demás grupos. Las redes que resultaron menos similares fueron las producidas por los grupos de mujeres adolescentes y ancianas, y por los de hombres jóvenes y ancianos. 


\section{Discusión}

A través de la presente investigación, se pudo observar que los conceptos de violencia y agresión tienen elementos compartidos y es por eso que en su manejo se presentan como sinónimos. Sin embargo, a través de los diferentes análisis permitidos por la técnica de Redes Semánticas Naturales Modificadas se encontró que ambos conceptos tienen elementos que permiten diferenciarlos. Más aún, se pueden identificar distintas concepciones de los estímulos con base en los distintos grupos de edad y sexo, ya que como se evidenció en los resultados, cada término varía según si se es adolescente, joven, adulto o anciano, y si se es hombre o mujer. Cada grupo incorpora elementos particulares que permiten que los conceptos adquieran matices y ocasionan que los términos violencia y agresión sean ambiguos en cuanto a lo que delimitan.

De hecho, tales términos son frecuentemente utilizados como sinónimos; sin embargo, a pesar de compartir algunos puntos en común en significado, no son idénticos. En la literatura científica la violencia se define como actos cometidos con la intención de infligir daño físico a alguien o algo, mientras que la agresión se le concibe como una conducta que intenta dañar en lo físico o en lo psicológico.

Al considerar que las diferentes teorías y clasificaciones de la agresión son más comunes en Psicología que las de violencia, se decidió basar las comparaciones entre estos dos conceptos a partir de los enfoques de la agresión. A lo largo de los estudios realizados se consideró importante evaluar el papel que juegan algunas variables de carácter demográfico en la representación social de la violencia y la agresión, tales como la edad (Forgas, Brown, \& Menyhart, 1980) y el sexo (Campbell, Sapochnik, \& Muncer, 1997; Campbell, Muncer, \& Gorman, 1993; Harris \& Knight- Bohnoff, 1996a; Harris \& Knight- Bohnoff, 1996b).

Como lo refiere McLellan (2002), se necesitan más estudios que se enfoquen en la concepción de los tipos de violencia específicos para las diferentes culturas, ya que, justamente, el entendimiento de lo que se consideran conductas aceptables pro- vienen del contexto sociocultural de cada país. Siguiendo esta idea se decidió que para alcanzar los objetivos definidos para la investigación, se requerían dos estudios que permitieran definir a la violencia y la agresión, así como identificar sus dimensiones. Se identificaron las diversas palabras que se asocian con la violencia y la agresión, lo que permitió poner en claro el significado psicológico de cada uno de estos conceptos, siguiendo los lineamientos de la técnica de Redes Semánticas Naturales Modificadas (Reyes-Lagunes, 1993).

Como Miller lo indica (citado por Cacciari \& Levorato, 2000), las palabras representan conceptos que las personas han encontrado lo suficientemente útiles y que ameritan lexicalización, por otro lado, estos conceptos juegan un papel importante en el sistema de creencias que la gente comparte. Se observa, a través del estudio que, efectivamente, ambos conceptos, violencia y agresión, tienen elementos compartidos y es por esa razón que en su manejo coloquial se muestran como sinónimos. La violencia con base en el número de definidoras reportadas es el concepto más ambiguo, lo que apoya la noción de fenómeno complejo y poco delimitado (Signorielli, Gerbner \& Morgan, 1995).

Como se puede consultar en el marco teórico de esta investigación ${ }^{1}$, resulta un trabajo muy laborioso identificar la diferencia entre agresión y violencia y aún más difícil es circunscribir cuál de las dos engloba a la otra. En este estudio se pudo establecer que ambas son muy similares, pero la agresión resulta más conductual y más emocional que la violencia. Ésta es más general y, sobre todo, circunscribe varios actos agresivos, mientras que agresión hace referencia a aspectos más individuales.

A diferencia de las definiciones etimológicas no se asocia a la violencia, como concepto psicosocial, al poder y la fuerza, lo que quiere decir que el concepto psicológico se parece más a la agresión que a su definición latina. La impetuosidad o arrebato que trae consigo el término tampoco está asociado con el significado, ya que en nin-

\footnotetext{
1 Para acceder a esta información se debe contactar a la autora.
} 
guna red semántica se obtuvieron palabras que denotaran este efecto.

Retomando algunas de las clasificaciones desarrolladas por Buss (1963); Geen (1968); Lorenz (citado por García \& Martínez, 1989); Zillman (1988) se observa que existen diferencias entre los conceptos de violencia y agresión. En muchas ocasiones la definidora guerra que se encuentra en el núcleo de violencia no obedece al ímpetu, sino a la instrumentación o al ejercicio del poder, lo mismo que los robos y asaltos, a diferencia de las definidoras asociadas a agresión, que obedecen a naturaleza más emocional como la rabia y la ira, lo que hace una distinción muy importante. Ambos conceptos, violencia y agresión engloban, en mayor número, conductas $\mathrm{y}$, como lo señala Buss (1966), estas acciones pueden ser de tipo físico o verbal. En las dos redes semánticas globales se encuentran más asociadas las conductas de tipo verbal a agresión que a violencia, como lo son las groserías, insultos y ofensas.

En la literatura se han asociado diversas variables a episodios de agresión y violencia, como por ejemplo, el alcohol, las armas y las drogas, pero estas variables no se identificaron dentro de los NR, con excepción de armas para adolescentes y adultos, y drogas para hombres, en la red de violencia; sin embargo, sí se encuentran en menor medida a lo largo de las redes y la asociación varía según el grupo de edad y sexo. Aunque otras variables medioambientales y biológicas se han identificado como agentes dentro del fenómeno de la agresión, no se asocian con el significado psicosocial de ninguno de los dos conceptos en el presente estudio.

El NR es el núcleo central del que hablan Wagner, Elejebarrieta y Valencia (1994) y es el que hace posible el entendimiento de un concepto, mientras que las definidoras que se encuentran fuera del NR son los elementos periféricos que son temporales. Retomando las palabras de Jodelet (2000), se puede observar en el estudio que las definidoras de las redes semánticas para cada concepto incorporan palabras semejantes, lo que hace posible que los individuos puedan entender o catalogar un evento como violento de manera muy similar. Sin embargo, como esta misma autora siguiere, existen cambios con el tiempo y por el efecto de otras variables, que hacen que la representación de un fenómeno tenga variantes. Es por esto que se encuentran en los resultados diferentes configuraciones en los núcleos de red, así como en las redes en su totalidad, lo cual obedece a la influencia del sexo y la edad.

Entre las limitantes de la investigación se encuentra el tipo de muestreo no probabilístico llevado a cabo, ya que algunos de los hallazgos no pueden ser generalizados, en ocasiones, por el tipo de institución en el que se recolectó la muestra. En el caso de los adolescentes y jóvenes, éstos fueron convocados en centros educativos de nivel medio y nivel superior, lo que implica un nivel de instrucción mínimo que, en el mejor de los casos, estaría representando un $10 \%$ de la totalidad de individuos que pueden ser incluidos en estos sectores. La mayoría de adultos de los grupos evaluados en esta investigación cuentan con un nivel universitario, por lo que el nivel educativo puede estar diferenciando a los grupos, y resulta una variable lo suficientemente fuerte como para que se considere en investigaciones posteriores.

Otra variable que se debe tener en cuenta en futuras investigaciones es el nivel socio-económico, ya que los fenómenos asociados a la violencia y la agresión, especialmente el maltrato y la violencia intrafamiliar, se presentan de manera diferenciada en los diferentes estratos, lo que impacta directamente los significados.

Finalmente, en Colombia, donde el conflicto armado ha permeado la historia durante más de cincuenta años, también sería interesante indagar sobre los significados en otros segmentos de la población, especialmente en áreas rurales, lo que permitiría tener una idea más clara de los fenómenos de interés.

\section{Referencias}

Adair, J. (1994). Perspectivas de la indigenización: diferentes aproximaciones al desarrollo de una psicología culturalmente propia. Re- 
vista de Psicología Social y Personalidad, $X$ (1), 37-44.

Buss, A.H. (1966). Instrumentality of Aggression, Feedback, and Frustation as Determinants of Physical Aggression. Journal of Personality and Social Psychology, 3, 153-162.

Buss, A.H. (1963). Physical Aggression in Relation to Different Frustations. Journal of Abnormal and Social Psychology, 67, 1-7.

Cacciari, C. \& Levorato, M.C. (2000). The Semantic Structure of Vision Verb: A Psycholinguistic Investigation of Italian. European Journal of Cognitive Psychology, 12 (1), 87-106.

Campbell, A., Muncer, S. \& Gorman, B. (1993). Sex and Social Representations of Aggression: A Communal-Agentic analysis. Aggressive Behavior, 19, 125-135.

Campbell, A., Sapochnik, M. \& Muncer, S. (1997). Sex Differences in Aggression: Does Social Representation Mediate form of Aggression? British Journal of Social Psychology, 36, 161-171.

Conrado, E.L. (1970). Violencia y estructuras. Buenos Aires, Argentina: Búsqueda.

Corsini, R.J. (1999). The Dictionary of Psychology. USA: Brunner-Mazel.

Domínguez, A. (2003). La violencia: su conceptuación y socialización a través de la televisión. Tesis de doctorado no publicada, Universidad Nacional Autónoma de México, México.

Figueroa, N; González, E. \& Solís, V. (1981). Una aproximación al problema del significado: las redes semánticas. Revista latinoamericana de Psicología, 13 (3), 447-458.

Forgas, J. P., Brown, L.L. \& Menyhart, B. (1980). Dimensions of Aggression: the Perception of Aggressive Episodes. British Journal of Social and Clinical Psychology, 19, 215-227.
García, J.F. (27 de septiembre de 1999). Sociedad y violencia. Trabajo presentado en el Panel sobre Sociedad y Violencia. Instituto Tecnológico de Santo Domingo. Recuperado el 4 de abril de 2000 de: http://areasociales/sociedad/perpectiva_judicial.html.

García, J. \& Martínez, J. (1989). Algunas perspectivas teóricas acerca de la agresión. Tesis de licenciatura no publicada, Universidad Nacional Autónoma de México, México.

Geen, R.G. (1968). Effects of Frustration, Attack, and Prior Training in Aggressiveness Upon Aggressive Behavior. Journal of Personality and Social Psychology, 9, 316-321.

Harris, M.B. \& Knight- Bohnoff, K. (1996a). Gender and Aggression I: Perceptions of aggression. Sex Roles, 35, 1/2, 1-25.

Harris, M.B. \& Knight-Bohnoff, K. (1996b). Gender and Aggression II: Personal Aggressiveness. Sex Roles, 35, 1/2, 27-42.

Holtzman, W. (1994). en busca de un lenguaje común en la medición psicológica y psiquiátrica: El Centro de Colaboración Texas- OMS. Revista de Psicología Social y Personalidad, $X$ (1), 45-58.

Jodelet, D. (2000). Representaciones sociales: contribución a un saber sociocultural sin fronteras. En D. Jodelet \& A. Guerrero (eds.). Develando la cultura. Estudios en representaciones sociales. México: Universidad Nacional Autónoma de México.

McLellan, F. (2002). Do Violent Movies Make Violent Children? Lancet, 359, 9305, 502.

Moscovici, S. (1984). Psicología Social II: Pensamiento y vida social. Psicología social y problemas sociales. Barcelona: Paidós.

Osgood, C. (1952). The Nature and Measurement of Meaning. Psychological Bulletin, 49 (9), 197-237. 
Real Academia Española. (2007). Gran Diccionario de la Lengua Española. Barcelona: Larousse Planeta S.A.

Reyes-Lagunes, I. (1993). Redes semánticas para la construcción de instrumentos. Revista de Psicología Social y Personalidad, IX (1), 83 -99

Rosario, R.C. (27 de septiembre de 1999). Discurso de Bienvenida al Panel sobre Sociedad y Violencia. Instituto Tecnológico de Santo Domingo. [4 de abril de 2000]

Salas-Menotti, I. (2003). El significado psicológico de la justicia, la igualdad y la equidad: Un estudio transcultural entre México y Colombia. Revista de Psicología Social y Personalidad, 19 (1), 1-20.

Signorielli, N., Gerbner, G. \& Morgan, M. (1995). Violence on Television: The Cultural Indicators Proyect. Journal of Broadcasting \& Electronic Media, 39, 278-283.
Szalay, L. \& Bryson, J. (1974). Psychological Meaning: Comparative Analyses and Theoretical Implications. Journal of Personality and Social Psychology, 30 (6), 860-870.

Szalay, L. \& Bryson, J. (1973). Measurement of Psychocultural Distance: A Comparison of Americans Blacks and Whites. Journal of Personality and social Psychology, 26 (2), 166-177.

Wagner, W., Elejabarrieta, F. \& Valencia, J. (1994). Estabilidad en las representaciones sociales de paz y guerra en dos países. Revista de Psicología Social y Personalidad, 10 (2), 123-143.

Zillman, D. (1988). Cognitive-Excitation Interdependencies in Aggressive Behavior. Aggressive Behavior, 19, 51-64.

Valdez, J.L. (1998). Las redes semánticas naturales, usos y aplicaciones en Psicología Social. Toluca: Universidad Autónoma del Estado de México. 